\title{
PROSPECÇÃO DA DIAPAUSA DA MARIPOSA-ORIENTAL NO PERÍODO DE DORMÊNCIA DO PESSEGUEIRO
}

\author{
PROSPECT ON DIAPAUSE OF THE ORIENTAL FRUIT MOTH IN THE \\ DORMENT PERIOD OF THE PEACH TREE
}

\author{
Alex Sandro POLTRONIERI ${ }^{1}$ \\ Lino Bittencourt MONTEIRO2 \\ Joselia Maria SCHUBER ${ }^{3}$
}

\begin{abstract}
RESUMO
Com o objetivo de verificar a existência de diapausa da mariposa-oriental durante a dormência do pessegueiro, foram monitorados adultos e lagartas de G. molesta em quatro pomares de pessegueiro 'Chimarrita', entre abril e agosto de 2006, em Araucária, PR. O monitoramento de adultos foi feito por meio de duas armadilhas Delta por pomar, e próximo a um dos pomares foram instaladas duas armadilhas na mata, distantes $100 \mathrm{~m}$ dos pessegueiros, para verificar a ocorrência de capturas do inseto fora do pomar. O monitoramento de lagartas foi feito com cintas-armadilhas, instaladas em trinta plantas por pomar, e em plantas que poderiam atuar como hospedeiras alternativas do inseto, além da realização de análises visuais nas reentrâncias dos troncos e ramos primários. As armadilhas Delta revelaram a ocorrência de adultos da mariposa-oriental nos pomares no período de dormência do pessegueiro, entretanto, nas armadilhas localizadas na mata, não ocorreram capturas. Não foram encontradas lagartas de G. molesta nas cintas-armadilha, bem como nas análises visuais nos pessegueiros e nas plantas que poderiam atuar como hospedeiras alternativas.
\end{abstract}

Palavras-chave: Grapholita molesta; lagartas; flutuação populacional.

\begin{abstract}
In order to observe the diapause of the oriental fruit moth during the dormant period of peach trees, G. molesta adults and caterpillars were monitored in four 'Chimarrita' peach orchards, between April and August 2006, in Araucária, PR, Brazil. Adults were monitored with two Delta traps per orchard; in addition, two traps were installed in a forest patch near one of the orchards, located $100 \mathrm{~m}$ away from the peach trees, to observe the occurrence of moth catches out of the orchard. Caterpillars were monitored with strip traps installed in thirty plants per orchard, and in plants that could serve as alternative hosts for the insect; also, the recesses on the trunks and primary branches were inspected visually. The Delta traps revealed the occurrence of oriental fruit moth adults in the orchards during the peach dormancy period; however, no captures occurred in traps placed in the forest. No G. molesta caterpillars were found in the strip traps or in the visual analyses of peach trees and plants that could serve as alternative hosts.
\end{abstract}

Key-words: Grapholita molesta; caterpillars; population fluctuation.

\footnotetext{
${ }^{1}$ Engenheiro Agrônomo, aluno de mestrado do Programa de Pós-Graduação em Agronomia, Departamento. de Fitotecnia e Fitossanitarismo, CCA/UFPR, Curitiba, PR. Bolsista Capes. Rua Osmário de Lima, 578. Bairro Capão da Imbuia, Curitiba - PR, Email: alex.poltronieri@yahoo.com.br. Autor para correspondência.

${ }^{2}$ Engenheiro Agrônomo, Dr. Professor do Programa de Pós-Graduação em Agronomia, Departamento de Fitotecnia e Fitossanitarismo, CCA/ UFPR, Curitiba, PR. Email: Ibmonteiro@terra.com.br

${ }^{3}$ Engenheira Agrônoma, aluna de mestrado do Programa de Pós-Graduação em Agronomia, Departamento de Fitotecnia e Fitossanitarismo, CCA/UFPR, Curitiba, PR. Bolsista CNPq. Email: joseliaschuber@yahoo.com.br
} 


\section{INTRODUÇÃO}

A mariposa-oriental, Grapholita molesta (Busck, 1916) (Lepidoptera: Tortricidae) é uma praga polífaga (GONZALEZ, 2003) que causa danos às frutíferas da família Rosaceae, destacando-se o pessegueiro, a macieira, a pereira, ameixeira e o marmeleiro (ALLEN e PLASKET, 1958; SALLES, 2000; MYERS et al.; 2006), não havendo registros no Brasil de plantas silvestres que atuem como hospedeiras do inseto (SALLES, 2000).

A flutuação de adultos da mariposa-oriental ocorre na primavera e verão em regiões produtoras de pêssego, entretanto, o inseto entra em diapausa no outono e inverno nas regiões de clima temperado (OMELYUTA, 1978), pois as temperaturas nesse período são inferiores a sua temperatura de base $\left(8,99^{\circ} \mathrm{C}\right)$ (GRELLMANN et al., 1992).

No Brasil a presença de indivíduos em diapausa não foi devidamente demonstrada, embora HICKEL et al. (2003a) sustentem que ocorra diapausa hibernal não sincronizada para G. molesta, em função das oscilações térmicas, pois se verificam capturas durante o inverno, sugerindo a existência de atividade de adultos e possivelmente de lagartas. Neste contexto, apenas o monitoramento de adultos não forneceria subsídios para se determinar com precisão se há lagartas em diapausa, pois no sul do Brasil ocorrem condições para o desenvolvimento do inseto durante o ano todo (SALLES, 2000; MONTEIRO e HICKEL, 2004).

Nos pomares de pessegueiro, as maiores populações de $G$. molesta ocorrem entre dezembro e fevereiro (AFONSO et al., 2002; HICKEL et al., 2003a), períodos de altas temperaturas e disponibilidade alimentar. A partir de março, devido à redução da temperatura, ocorre a lignificação das brotações, acarretando em uma diminuição na oferta alimentar, o que afeta o desenvolvimento das lagartas de G. molesta (SOUZA et al., 2000).
Em macieiras ARIOLI (2007) constatou a presença de lagartas da mariposa-oriental alimentando-se de "Burrknots", presumindo que estas raízes aéreas sejam a única fonte alimentar do inseto nos pomares durante a primeira geração pós-diapausa. Em hipótese, os "Burrknots" poderiam atuar como abrigo e fonte alimentar para $o$ inseto durante o inverno.

Em pessegueiros não são encontradas estruturas de proteção e alimentação (Burrknot) para as lagartas passarem o inverno, como ocorre em macieiras. AUDEMARD (1976) e CHARMILLOT et al. (1997) procurando simular um abrigo hibernal para lagartas, instalaram cintas-armadilhas na base das árvores. Os tortricídeos alojam-se nas cintasarmadilhas, de modo que é possível realizar o monitoramento das lagartas analisando as mesmas.

O objetivo deste trabalho foi verificar a presença de adultos e lagartas de G. molesta em pomares de pessegueiro durante o período de dormência das plantas, bem como verificar a presença do inseto em plantas que poderiam atuar como hospedeiras alternativas.

\section{MATERIAL E MÉTODOS}

O estudo foi realizado entre $1^{\circ}$ de abril a 30 de agosto de 2006, em quatro pomares de pessegueiros 'Chimarrita', localizados em Araucária, Paraná $\left(25^{\circ} 35^{\prime}\right.$ de latitude sul, $49^{\circ} 24^{\prime}$ de longitude oeste e $897 \mathrm{~m}$. de altitude), durante o período de dormência, caracterizado pela ausência de folhas e lignificação das brotações das plantas. A idade dos pomares variou de 6 a 12 anos, sendo conduzidos em taça com quatro ramos primários, com uma área média de 0,36 ha, o que corresponde ao tamanho médio dos pomares desta frutífera no município (Tabela 1).

TABELA 1 - Caracterização dos pomares, cultivar Chimarrita, utilizados para o estudo do desenvolvimento de Grapholita molesta durante o período de dormência do pessegueiro em Araucária, PR. Abril/ Agosto de 2006.

\begin{tabular}{cccccc}
\hline Pomar & Idade (anos) & Área (ha) & Espaçamento $(\mathrm{m})$ & $\mathrm{N}^{\circ}$ de linhas & $\mathrm{N}^{\circ}$ de plantas \\
\hline A & 6 & 0,30 & $6 \times 4$ & 4 & 123 \\
B & 8 & 0,30 & $6 \times 4$ & 3 & 120 \\
C & 10 & 0,48 & $6 \times 8$ & 6 & 100 \\
D & 12 & 0,37 & $6 \times 4$ & 11 & 185 \\
\hline
\end{tabular}

\section{Monitoramento da atividade de adultos de $G$. molesta}

A flutuação populacional de adultos de $G$. molesta foi obtida por meio de duas armadilhas Delta por pomar, contendo um septo de feromônio sexual marca Biographolita ${ }^{\circledR}$, Biocontrole Ltda, São Paulo, SP. As armadilhas foram instaladas na terceira linha de plantio das bordaduras, na parte externa da planta a $1,70 \mathrm{~m}$ de altura, em um ramo primário voltado para o poente. Os septos de feromônio foram trocados em intervalos de seis semanas e o piso adesivo foi substituído quando havia necessidade (ARIOLI et al., 2005). A inspeção das armadilhas foi realizada semanalmente, sendo os insetos capturados, contados e retirados.

Para verificação da ocorrência de G. molesta fora do pomar, instalaram-se duas armadilhas Delta em mata ciliar, situada a 100 metros do pomar. Este estudo foi realizado apenas no pomar ' $\mathrm{C}$ ', por possuir 
a maior bordadura com a mata, entre os quatro pomares acompanhados.

\section{Monitoramento de lagartas de G. molesta nos pomares}

O monitoramento de lagartas foi feito por meio da instalação de cintas-armadilha e por amostragens visuais nas reentrâncias dos troncos e ramos primários. Foram selecionadas 30 plantas, as quais foram colocadas as cintas-armadilha constituídas por tiras de papelão ondulado, perfil de onda $B(2,3$ a $2,6 \mathrm{~mm})$, com $10 \mathrm{~cm}$ de largura e comprimento suficiente para dar duas voltas ao redor do tronco da planta. As cintas-armadilha foram instaladas entre 10 e $15 \mathrm{~cm}$ do solo, de acordo com a metodologia proposta por MOLINARI (1942). As avaliações visuais nas reentrâncias foram feitas nos quatro ramos primários e troncos dos pessegueiros selecionados, de modo que em cada planta foram gastos aproximadamente 5 minutos de vistoria. Ambas as amostragens foram realizadas quinzenalmente, e as pupas e/ou lagartas encontradas foram transferidas para um saco de tuly, permanecendo na própria cinta-armadilha para o monitoramento da emergência da mariposa.

\section{Monitoramento de lagartas de G. molesta em possíveis plantas hospedeiras alternativas}

A presença de G. molesta, em plantas que poderiam atuar como hospedeiras foi analisada em um perímetro de $100 \mathrm{~m}$ ao redor de cada pomar, identificando as árvores frutíferas e/ou nativas entre as mais representativas e aquelas que compunham os quebra-ventos (Tabela 2). As amostragens foram feitas instalando cintas-armadilha, similares as instaladas nos pessegueiros, e o monitoramento visual em troncos e ramos. Em frutíferas foram realizadas amostragens visuais em 100 frutos. Quando presentes, os frutos danificados eram coletados e levados para o Laboratório de Manejo Integrado de Pragas (Lamip), do Departamento de Fitotecnia e Fitossanitarismo, do Setor de Ciências Agrárias, da UFPR, para verificação da presença do inseto e confirmação da espécie. As avaliações de cintas-armadilha, troncos e frutos foram feitas a cada quinze dias.

TABELA 2 - Espécies vegetais avaliadas para verificar a presença de Grapholita molesta em um perímetro de $100 \mathrm{~m}$ dos pomares de pessegueiro 'Chimarrita' em Araucária, PR. Abril/ Agosto de 2006.

\begin{tabular}{ccccc}
\hline Pomar & Planta & Família & No de Plantas & Análise (100 Frutos) \\
\hline A & Cedrinho (Cedrus deodora) & Cupresaceae & 3 & Não \\
A & Jerivá (Syagrus romanzoffiana) & Arecaceae & 1 & Não \\
A & Maracujá (Passiflora edulis) & Passifloraceae & 1 & Sim \\
A & Nêspera (Eribotrya japonica) & Rosaceae & 1 & Sim \\
B & Araçá (Psidium araçá raddi) & Myrtaceae & 2 & Sim \\
B & Cedrinho (Cedrus deodora) & Cupresaceae & 2 & Não \\
B & Laranja (Citrus x sinensis) & Rutaceae & 4 & Sim \\
B & Pinheiro Bravo (Pinus pinaster) & Pinaceae & 2 & Não \\
C & Jabuticaba (Plinia trunciflora) & Mirtaceae & 1 & Sim \\
C & Pinheiro (Araucaria augustifolia) & Araucariaceae & 4 & Não \\
D & Caqui (Diospyros kaki) & Ebenaceae & 4 & Sim \\
D & Jabuticaba (Plinia trunciflora) & Mirtaceae & 1 & Sim \\
D & Morgota (Citrus reticulata) & Rutaceae & 3 & Sim \\
D & Nêspera (Eribotrya japonica) & Rosaceae & 1 & Sim \\
D & Pêra (Pírus calleryana) & Rosaceae & 1 & \\
\hline
\end{tabular}

\section{Estimativa do número de gerações de G. molesta no período de dormência do pessegueiro}

A estimativa do número de gerações foi calculada entre abril e agosto de 2006, utilizando a constante térmica de 480 graus-dia (GD), necessária para que o inseto complete seu ciclo de desenvolvimento (GRELLMANN et al., 1992), e a soma de graus-dia (GD), baseando-se na fórmula descrita por SILVEIRA NETO et al. (1976), conforme as seguintes situações:

a) Temperatura mínima diária maior que a TB do inseto $\left(8,99^{\circ} \mathrm{C}\right)$ :

GD = ((t.máx. + t.mín.) /2) - TB

b) Temperatura mínima diária menor que a TB do inseto $\left(8,99^{\circ} \mathrm{C}\right)$ :

$$
\mathrm{GD}=(\text { t.máx. }+\mathrm{TB})^{2} / 2 \text { (t.máx. - t.mín.) }
$$

Onde: $\quad \mathrm{GD}=$ Graus-dia; T.máx = temperatura máxima $\left({ }^{\circ} \mathrm{C}\right) ;$ T.mín. = temperatura mínima $\left({ }^{\circ} \mathrm{C}\right)$; TB $=$ temperatura-base $\left({ }^{\circ} \mathrm{C}\right)$.

Os dados climáticos utilizados para a região de Araucária, PR, foram fornecidos pelo SIMEPAR (Instituto Tecnológico Sistema de Informações Meteorológicas do Paraná).

\section{RESULTADOS E DISCUSSÃO}

\section{Monitoramento de adultos de G. molesta}

Foram verificadas capturas de adultos de G. molesta durante todo o período de monitoramento, sendo que, entre abril e junho de 2006 ocorreu o maior número de indivíduos em detrimento a julho e agosto de 2006 (Figura 1). 


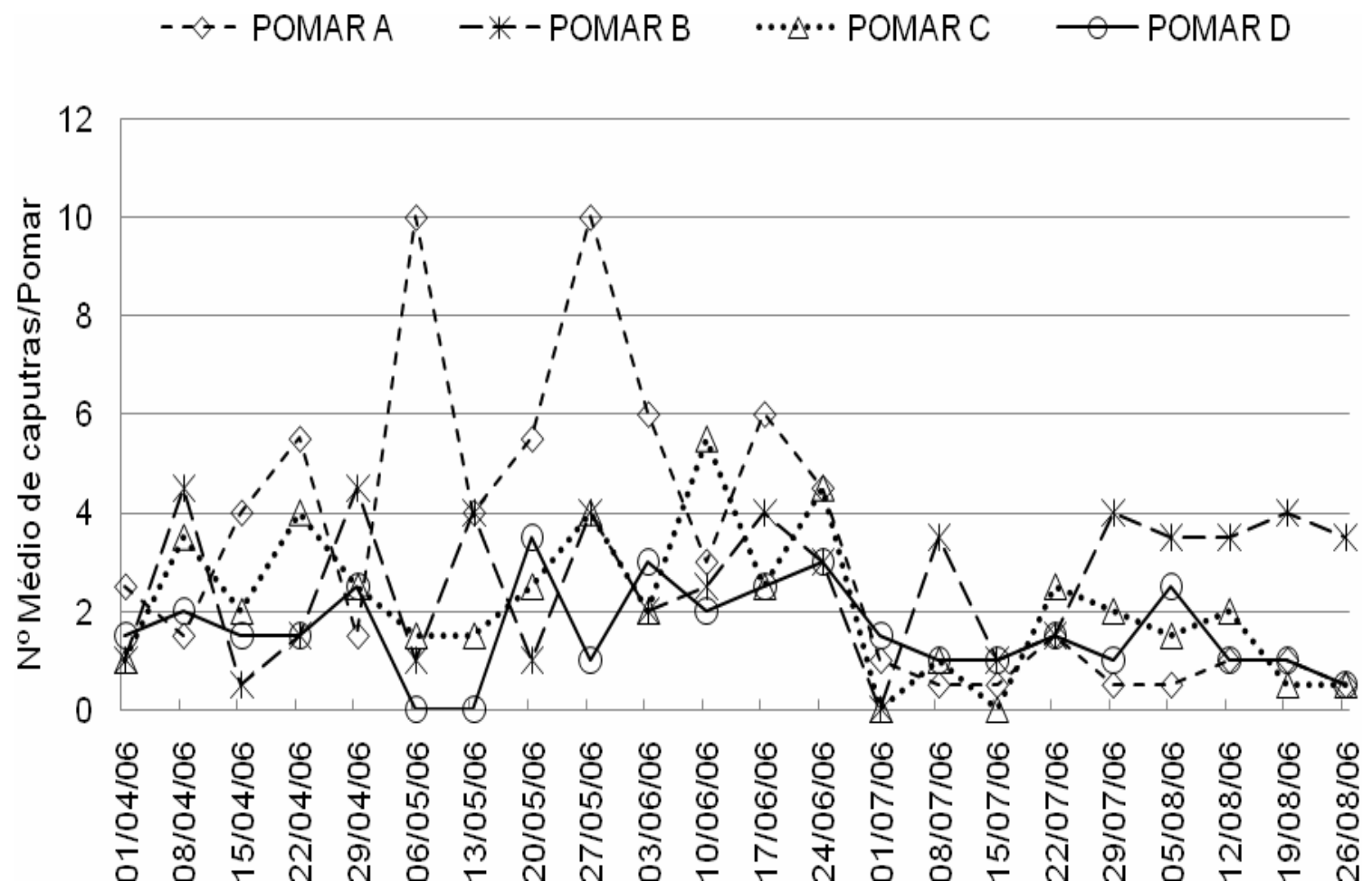

FIGURA 1 - Flutuação populacional de machos de Grapholita molesta capturados por meio de armadilhas Delta em quatro pomares de pessegueiro 'Chimarrita' em Araucária, PR. Abril a agosto de 2006.

A temperatura média registrada em Araucária, entre abril e agosto, foi de $15,9^{\circ} \mathrm{C}$ (Tabela 3), próxima aos $16{ }^{\circ} \mathrm{C}$ de citado por SALLES (2000) como a temperatura base para o vôo espontâneo de G. molesta e superiores as observadas nas regiões centrais do Chile, por GONZALEZ (2003). A captura da mariposa-oriental em Araucária ocorreu no período de dormência do pessegueiro, resultado concordante com os de SALLES (2000), que argumenta que as temperaturas registradas nas regiões produtoras de fruteiras de caroço do Brasil permitem ao inseto se desenvolver durante todo o ano. Entretanto, HICKEL, et al., (2003b) supõem que as capturas de adultos no período hibernal, se devem a falta de um perfeito sincronismo na diapausa entre os indivíduos. GONZALES (2003) mostrou que há ocorrência de adultos quando a temperatura média oscilou entre 7,5 e $8{ }^{\circ} \mathrm{C}$ e OMELYUTA (1978) quando esta era de $10^{\circ} \mathrm{C}$.

As armadilhas Delta que estavam localizadas na mata, a 100 metros do pomar C, não realizaram capturas de adultos de G. molesta, embora tenham sido registradas capturas neste pomar.

TABELA 3 - Média mensal das temperaturas máximas, médias e mínimas ocorridas entre abril e agosto de 2006. Acúmulo mensal de Graus-dia (GD), considerando-se a temperatura base para o desenvolvimento de Grapholita molesta $\left(8,99^{\circ} \mathrm{C}\right)$, no período de abril a agosto de 2006 em Araucária, PR.

\begin{tabular}{ccccc}
\hline \multirow{2}{*}{ Mês } & \multicolumn{3}{c}{ Temperatura média $\left({ }^{\circ} \mathrm{C}\right)$} & \multirow{2}{*}{ Acúmulo mensal (GD) } \\
\cline { 2 - 4 } & Máxima. & Mínima & Média & 354,5 \\
\hline Abril & 23,7 & 14,3 & 18,1 & 158,9 \\
Maio & 19,6 & 9,6 & 14,3 & 227,7 \\
Junho & 22,2 & 12,1 & 14,8 & 261,9 \\
Julho & 22,1 & 10,8 & 15,9 & 218,6 \\
Agosto & 22,3 & 10,9 & 16,2 & Soma GD 1221,6 \\
\hline Média & 22,0 & 11,5 & 15,9 &
\end{tabular}

Fonte: SIMEPAR (2006) 


\section{Monitoramento de lagartas de G. molesta nos pomares}

Não houve ocorrência de lagartas nas cintas-armadilha durante o período de monitoramento. Esperava-se que o inseto apresentasse preferência por empupar nas cintasarmadilha (MOLINARI, 1942), contudo isso não ocorreu. Em Mendonza, Argentina, o Instituto de Sanidad y Calidad Agropecuária Mendoza (ISCAMEN) (1999), recomenda as cintas-armadilha para o controle de lagartas de Cydia pomonella (Lepidoptera: Tortricidae) e G. molesta, visto que as lagartas que saem dos frutos ou ponteiros para empupar adotam as cintas-armadilha como sítios ou abrigos para se alojarem.

A ausência de lagartas nas cintasarmadilha poderia ser atribuída ao número de amostras e localização das mesmas, pois segundo ISCAMEN (1999), estas armadilhas devem ser instaladas em quase todas as plantas localizadas no pomar. Contudo, o objetivo dos estudos em Araucária foi de monitoramento, e nesse caso, a recomendação é de 1 a $2 \%$ do total das plantas (AUDEMARD, 1976). A pesquisa recomenda para áreas de 1 a 4 ha cerca de 30 a 40 cintas-armadilhas, e segundo BERS et al. (1993), as armadilhas devem ser instaladas nas duas primeiras linhas de bordadura do pomar, bem como em plantas situadas no seu interior. Em Araucária foram colocadas $50 \%$ de cintas-armadilhas na bordadura e $50 \%$ no interior do pomar.

A época de instalação das cintas-armadilha pode ter corroborado para que não ocorressem capturas, pois é recomendado que as armadilhas sejam instaladas em janeiro e que sejam verificadas a partir de maio (ISCAMEN, 1999). Assim, como os trabalhos foram iniciados em abril, em hipótese, é provável que os insetos tenham migrado para abrigos invernais antes da instalação das armadilhas.

A ausência de lagartas e/ ou pupas de $G$. molesta, verificada por meio das inspeções visuais realizadas nas plantas deixa dúvidas sobre a presença das mesmas em pessegueiro no período de dormência, mesmo havendo capturas de adultos em armadilha Delta. Diversos estudos, como os de HICKEL et al. (2003b) e HICKEL (2006), comentam que a $G$. molesta sobrevive ao inverno em diapausa na fase larval, sob a casca das árvores e em folhas aderidas a ramos, mas isso não foi verificado pelas inspeções visuais executadas. A cultivar Chimarrita não apresenta locais de abrigo para G. molesta como ocorre em macieiras com os "Burrknots", alojando lagartas de G. molesta ${ }^{1}$, portanto é provável que as lagartas estejam em outros locais no pomar.

Estimativa do número de gerações de G. molesta no período de dormência do pessegueiro

Quando se calcula os graus-dia necessários para desenvolvimento da mariposaoriental em Araucária, entre os meses de abril a agosto, constata-se um acúmulo de 1221,6 GD (Tabela 3), suficiente para haver duas gerações de G. molesta no período, indicando que cada geração levaria entre 76 e 77 dias para completar seu ciclo. Estes resultados são inferiores aos de BOBADILLA (1972) no Chile, onde foram necessários 120 dias de acúmulos de graus-dia para uma geração do inseto.

Monitoramento de lagartas de G. molesta em possíveis plantas hospedeiras alternativas

O monitoramento realizado em possíveis plantas hospedeiras alternativas fora dos pomares de pessegueiro, tanto por cintas-armadilhas como por inspeção visual nos ramos, não revelou a presença de lagartas da mariposa-oriental. Estes resultados estão de acordo com SALLES (1991) que desconhece as plantas que poderiam atuar como hospedeiros alternativos de G. molesta.

Nos frutos coletados não foram encontradas lagartas, resultados estes discordantes dos observados por SALLES (2000) e GONZALEZ (2003), que relataram a incidência de lagartas de $G$. molesta em nêspera (Eribotrya japonica).

A ausência de lagartas na parte aérea das plantas corrobora com os dados obtidos nas cintasarmadilha nos quatro pomares, o que sugere que novos estudos, nas condições do sul do Brasil, durante o período de dormência do pessegueiro, ainda são necessários para desvendar a localização e existência de diapausa em lagartas de G. molesta.

\section{CONCLUSÕES}

Não foi observada a presença de lagartas de G. molesta em pessegueiros e nas plantas nativas prospectadas como possíveis hospedeiras alternativas, durante o período de dormência do pessegueiro em Araucária, PR.

Há ocorrência de adultos de G. molesta nos pomares durante o período de dormência do pessegueiro, em Araucária, PR.

\footnotetext{
${ }^{1}$ Comunicação pessoal de Lino B. Monteiro (UFPR), observação realizada em Fraiburgo, SC.
} 


\section{REFERENCIAS}

1. AFONSO, A.P.S.; GRÜTZMACHER, A.D.; LOECK, A.E.; FACHINELLO, J.C.; HERPICH, M.I.; BECKMANN, M.Z. Flutuação populacional e danos de Grapholita molesta (Busck, 1916) (Lepidoptera: Tortricidae) em sistemas de produção convencional e integrada da cultura do pessegueiro na localidade de Pelotas, RS. Revista Brasileira de Agrociência, v. 8, n. 3, p. 225-229, 2002.

2. ALLEN, H.W.; PLASKET, E.L. Populations of the oriental fruit moth in peach and apple orchards in the eastern state. Washington, USDA, 1958. (Tech. Bull., 1182).

3. ARIOLI, J.C.; CARVALHO, G.A.; BOTTON, M. Flutuação populacional de Grapholita molesta (Busck) com armadilhas de feromônio sexual na cultura do pessegueiro em Bento Gonçalves, RS, Brasil. Ciência Rural, v. 32, n. 1, p. 1-5, 2005.

4. ARIOLI, J.C. Técnica de criação e controle de Grapholita molesta (Busck, 1916) (Lepidoptera: Tortricidae) na cultura da macieira. Pelotas, 2007. 100 f. Tese (Doutorado em Entomologia) - Universidade Federal de Pelotas.

5. AUDEMARD, H. Etude demoecologique du carpocapse (Lasperyresia pomonella L.) em vergens de pommier de la Basse Vallée du Rhone. Possiblilités d'organisation d'une lutte intégrée. Tours, 1976. 340 f. Tese (Doutorado em Agronomia - Entomologia) - Curso de Pós-graduação em Agronomia, Université de Tours.

6. BEERS, E. H.; BRUNNER, J. F.; WILLET, M. J.; WARNER, G. M. Orchard pest management. Washington: Good Fruit Grower, 1993. p. 65-66.

7. BOBADILLA, R. Polilla oriental de la fruta. Santiago: Servício Agrícola y Ganadero, 1972. (Boletin Técnico, 1952).

8. CHARMILLOT. P.J.; PASQUIER, D.; SCALCO, A.; HOFER, D. Lutte contre la carpocapsa Cydia pomonella L. par un procédé attracticide. Revue Suisse Viticulture, Arboriculture, Horticulture, v. 29, p. 111-117, 1997.

9. CIVIDANES, F.J.; MARTINS, I.C.F. Flutuação populacional e previsão de gerações de Grapholita molesta (Busck, 1916) (Lepidoptera: Tortricidae) em pomares de pessegueiro, Prunus persica (Linnaeus) Batsch. Acta Scientia Agronômica, v. 28 , n. 3, p. 399-405, 2006.

10. GONZALEZ, H.R. Las polillas de la fruta en Chile (Lepidoptera: Tortricidae; Pyralidade). Santiago: Universidad de Chile, 2003. 188 p. (Serie Ciências Agronomicas, 9).

11. GRELLMANN, E.O.; LOECK A.E.; SALLES, L.A.B., FACHINELLO, J.C. Necessidades térmicas e estimativa do número de gerações de Grapholita molesta (Busck, 1916) (Lepidoptera: Olethreutidae) em pelotas, RS. Pesquisa Agropecuária Brasileira, v. 27, n. 7. p. 999-1004, 1992.

12. HICKEL, E.R.; HICKEL, G.R.; DE SOUZA, O.F.F.; VILELA, E.; MIRAMONTES, O. Dinâmica populacional da mariposa oriental em pomares de pessegueiro e ameixeira. Pesquisa Agropecuária Brasileira, v. 38, n. 3, p. 325-337, $2003 a$.

13. HICKEL, E.R.; VILELA, E.; DE SOUZA, O.F.F. Previsão da atividade de vôo de Grapholita molesta (Busk) em pomares de pessegueiro e ameixeira, através do ajuste entre captura de adultos em armadilhas de feromônio e acumulação de calor. Revista de ciências Agroveterinárias, v. 2, n. 1, p. 30-41, 2003b.

14. HICKEL, E.R. Análise da ocorrência e decisão de controle da mariposa-oriental Grapholita molesta (Busk). In: ENCONTRO NACIONAL SOBRE FRUTICULTURA DE CLIMA TEMPERADO, 9., 2006, Fraiburgo. Anais (palestras). Caçador: EPAGRI, 2006. v. 1. p. 99-107.

15. INSTITUTO DE SANIDAD Y CALIDAD AGROPECUARIA MENDOZA - ISCAMEN. Programa lucha contra carpocapsa y grafolita. Mendoza, 1999. (Boletin técnico, 20). Disponível em: <http://www.iscamen.com.ar/capacitacion/ carpocapsa.pdf>. Acesso em: 21/12/2006.

16. LOECK, A.E.; ROSENTHAL, M. d' A.; GUSMÃO, L.G.; GRELLMANN, E.O. Ocorrência de parasitóides sobre lagartas de Grapholita molesta (Busck, 1916) na localidade de Pelotas, RS. Anais da Sociedade Entomológica do Brasil, v. 21 n. 2, p. 431-436, 1992.

17. MOLINARI, O.C. Entomologia agrícola: identificacion y control de insectos y otros animales dañinhos o utiles a las plantas. San Juan, 1942.

18. MYERS, C.T.; HULL, L.A.; KRAWCZYK, G. Comparative survival of oriental fruit moth (Lepidoptera: Tortricidae) larvae on shoots and fruit of apple and peach. Journal Economic Entomology, v. 99, n. 4, p. 1299-1309, 2006.

19. OMELYUTA, V.P. Effect of atmospheric temperature on the development of the oriental peach moth. Zakhist Roslin, v. 23, p. 3- 6, 1978.

20. SALLES, L.A.B. Grafolita (Grapholita molesta): bioecologia e controle. Pelotas: EMBRAPA-CNPFT, 1991.13 p. (EMBRAPA-CNPFT. Documentos, 42).

21. SALLES, L.A. Mariposa-oriental, Grapholita molesta (Lepidoptera: Tortricidae) In: VILELA, E.F.; ZUCCHI, R.A.; CANTOR, F. Histórico e impacto das pragas introduzidas no Brasil. Ribeirão Preto: Editora Holos, 2000. 173 p.

22. SILVEIRA NETO, S.; NAKANO, O.; BARBIN, D.; NOVA, N.A.V. Manual de ecologia dos insetos. São Paulo: Agronômica Ceres, 1976. 419 p.

23. SOUZA, B.; SANTA-CECÍLIA, V.C.; SOUSA, O.V. Ocorrência de danos de Grapholita molesta (Busck) (Lepidoptera: Tortricidae) em pessegueiros no município de Caldas, MG. Anais da Sociedade Entomológica do Brasil, v. 29, n. 1, p. 185-188, 2000. 
